\title{
Un poème de circonstance, le récit de bataille en vers pendant les guerres de religion (1580-1601)
}

\section{Bruno Méniel}

\section{(2) OpenEdition}

1 Journals

Édition électronique

URL : http://journals.openedition.org/studifrancesi/8802

DOI : 10.4000/studifrancesi.8802

ISSN : 2421-5856

Éditeur

Rosenberg \& Sellier

\section{Édition imprimée}

Date de publication : 1 octobre 2008

Pagination : 383-395

ISSN : 0039-2944

\section{Référence électronique}

Bruno Méniel, « Un poème de circonstance, le récit de bataille en vers pendant les guerres de religion (1580-1601) », Studi Francesi [En ligne], 155 (LII | II) | 2008, mis en ligne le 30 novembre 2015, consulté le 12 janvier 2021. URL : http://journals.openedition.org/studifrancesi/8802 ; DOI : https://doi.org/ 10.4000/studifrancesi.8802

\section{(c) (i) $\odot$}

Studi Francesi è distribuita con Licenza Creative Commons Attribuzione - Non commerciale - Non opere derivate 4.0 Internazionale. 


\section{Un poème de circonstance, le récit de bataille en vers pendant les guerres de religion (1580-1601)}

Les plus grandes œuvres de la poésie épique, de l'Iliade à la Chanson de Roland, semblent trouver leur origine dans un récit de bataille. Un examen attentif des poèmes qui se donnent pour première fonction de raconter une victoire militaire pourrait permettre de saisir le genre épique dans son surgissement, de l'appréhender sous sa forme native et archétypale. Or, en France, la période des guerres de religion a favorisé l'essor, au sein de la poésie épique, du poème de combat, qui raconte des événements récents et exprime une position politique et confessionnelle ${ }^{1}$. Les poètes de la fin du XVI siècle qui exploitent cette veine peuvent se réclamer de l'exemple antique de Lucain, dont ils se sentent proches en raison de l'analogie entre les guerres civiles romaine et française, mais aussi de l'expansion du néo-stoïcisme.

La poésie de la fin du XVI siècle est assez riche en récits de bataille. Nous avons limité notre investigation à une forme précise, celle du poème épique, c'est-à-dire d'un poème non strophique relativement long, en vers isométriques longs à rimes plates, de style élevé, comportant une proposition, une invocation, une dédicace et une narration. Nous avons écarté les œuvres intitulées «odes», «hymnes», «chants» ou «cantiques», termes indiquant l'appartenance à des genres particuliers. Ces trois critères - le récit de bataille, la forme épique, l'absence de détermination générique autre - permettent de constituer un corpus de neuf textes, en général composés peu après l'événement qu'ils relatent ${ }^{2}$.

Nous avons abordés ces textes en partant de la notion de «poème de circonstance». Bien sûr, cette notion critique est apparue dans l'histoire littéraire après la Re-

(1) Sur le poème de combat, voir notre Renaissance de l'épopée. La poésie épique, en France, de 1572 à 1623, Genève, Droz («Travaux d'Humanisme et Renaissance»), 2004, pp. 313-319.

(2) Les neuf textes sont les suivants: [anonyme,] L'Argument de la victoire que le Roy s'est acquise sur les Ristres, Paris, Jean Richer, 1588, in- $8^{\circ}$, p. 16; Michel de La ValleE du MaIne, Triplimachie, ou triple combat de l'union, Paris, Jean Durant, 1589 , 20 f.; Alexandre de Pontaymeri, La Cité de Montelimar ou les trois prinses d'icelle composees et redigees en sept livres, s.1., 1591, p. 252; [abbé Nicolas DE Montreux], La Miraculeuse Délivrance de Monseigneur le Duc de Guise, Nantes, Nicolas des Marestz et François Faverye, 1591, in-4; ID., L'beureuse et entiere victoire, obtenuë sur les ennemis de Dieu à Cran, par le grand, victorieux, et Catholique Prince, Philippes Emanuel de Lorraine [...], Nantes, Nicolas des Marestz et François Faverye, 1592; Sébastien GARNIER, Les Huict Derniers Livres de la Henriade [IX-XVI], contenans les faicts merveilleux de Henry, roy de France et de Navarre [...], Bloys, Vve B. Gomet, 1593 , in-4, VI f., p. 148, V f.; ID., Les Huict Premiers Livres de la Henriade, contenans les faits admirables de Henry roy de France [...], [ne comprend que les livres I-II], Bloys, Gomet, 1594 , in $-4^{\circ}$, XII f.- p. 39; [Jean-François Le Petit], La Miraculeuse Deffaite de l'armade navale, escriée invincible de Philippe d'Austrice, Roy de Castille, pretendant envabir le Royaume d'Angleterre ez Mois de Iuillet, Aoust et Septembre, l'An bisextil M.D.LXXXVIII, à la suite des Sonets des grans exploicts victorieux de bault et puissant seigneur Maurice, Prince d'Orange, Comte de Nassau [...] par I.F.L.P.G.D.B., s.1., 1598, in- $8^{\circ}$, f. Eij r ${ }^{\circ}-\mathrm{H} \mathrm{r}^{\circ}$; Jean BosQueT, Reduction de la ville de Bone, par Messire Charles, Duc de Croy et d'Arschot, Prince de Chimay, etc, en l'an 1588. Et autres siens faits mémorables, meslangés de succinct recit de plusieurs choses notables advenües depuis ledit an jusques à ce jour [...], Anvers, Martin Nutius, 1599, in-4 ${ }^{\circ}, 84$ f.; Pierre DE DeImier, L'Austriade, Lyon, Th. Ancelin, 1601, 12 f., p. 313. 
naissance. Elle nous semble cependant particulièrement adaptée ici. En effet, le critique Predrag Matvejevitch propose du mot «circonstance» l'élucidation suivante:

L'étymologie du mot circonstance renvoie à des choses ambiantes et extérieures (circum stantia). Ce mot comporte en outre - de même que les termes plus ou moins synonymiques comme occasion, cas, occurrence ou situation - l'idée d'une détermination temporelle. Ces caractéristiques se retrouvent également dans la poésie de circonstance qui comporte un lien plus ou moins direct avec l'extériorité et se présente dans un rapport immédiat avec la circonstance [...].

Une œuvre de circonstance (poétique, aussi bien que tout autre) peut être faite:

a) en vue d'un événement dont l'échéance est connue ou fixée à l'avance par quelque convention;

b) par rapport à des événements accomplis dans un passé plus ou moins éloigné et ayant quelque relation avec le moment présent ${ }^{3}$.

Or la bataille est l'événement par excellence, un événement extérieur, objectif et historique, et les récits en vers que nous considérerons sont bien composés par rapport à elle. Dans l'expression «poème de circonstance», le mot «circonstance» ne règle cependant pas la question du rapport entre le poème et l'événement: celui-ci peut être le «stimulant», le «point de départ», le «prétexte» de la création poétique ${ }^{4}$; mais, en sens contraire, le poème peut être ce qui constitue la bataille en événement et lui confère une dimension historique et même métaphysique.

D'emblée une tension apparaît entre l'objet et la forme, entre l'exiguïté spatiale et temporelle de l'événement et l'ampleur inhérente à l'epos. Notre hypothèse est que cette tension n'est pas appréhendée par les poètes comme une inadéquation de la forme à la matière, mais comme la source d'effets voulus, permettant aux poèmes de remplir des fonctions déterminées. De l'éclairage que donnent ces poèmes à l'événement nous tenterons de déduire les objectifs visés. Nous décrirons d'abord le dispositif, puis nous analyserons le rapport que ces poèmes instaurent avec l'espace et le temps, enfin nous déterminerons les fonctions du poème.

\section{Signification d'un dispositif}

Dans ces œuvres, le poète se place dans une situation d'énonciation précise et particulière. Il parle d'une bataille récente, d'ordinaire une victoire du camp auquel il appartient. Parfois même il a été témoin de l'événement; il a en tout cas recueilli des informations de première main. Il écrit à chaud. Il dédie son poème au vainqueur $\mathrm{du}$ jour. Nous avons inclus dans notre choix deux poèmes publiés assez longtemps après l'événement qu'ils relatent. La Reduction de la ville de Bone, publiée par Jean Bosquet à Anvers en 1599, raconte la prise de Bonn par le duc de Cray en 1588, mais le cadre du récit dépasse largement celui de l'événement, comme l'indique la dédicace aux gouvernants du Hainaut: «C'est, Messeigneurs, un poëme, mais plustost un tableau, dans lequel se voyent veritablement depeintes les actions, fortunes bonnes et sinistres, et les accidens divers, qui ont couru puis douze ans par la genereuse, meure, et prudente virilité de sadite Excellence»'. La mort du duc constitue l'événement récent provoquant l'émotion génératrice du poème. L'Austriade de Pierre de Deimier, imprimée en 1601, raconte la bataille de Lépanthe (1571), mais elle s'adresse à Char-

(3) P. Matvejevitch, Pour une poétique de l'événement, Paris, Union Générale d'Edition («10/18»), 1979, pp. 180-181; c'est l'auteur qui souligne.

(4) Ibidem, p. 217.
(5) J. Bosquet, 1599, f. *2 $\mathrm{r}^{\circ}-\mathrm{v}^{\circ}$. Les références complètes aux poèmes cités figurent dans la note 2 . 
les-Emmanuel de Savoie qui, s'il n'y a pas été présent, a envoyé des navires contre les Turcs et a même perdu un cousin au cours de l'affrontement. Le poète s'adresse au souverain en ces termes:

[...] vos valeurs qui donnent de l'envie à Mars, et de l'admiration aux mesmes merveilles, vous aquierent cest heroïque ouvrage par merite et mon ame qui n'a jamais admiré que la gloire, vous le vien offrir par devoir et par devotion. Aussi, en ce combat signalé des plus grands efforts de guerre, vos honneurs se vendiquent une partie des plus belles Palmes, puis que les genereux guerriers qui estoient portez sus vos Galeres si fameuses y feirent si bravement de courage et de force ${ }^{6}$.

Charles-Emmanuel a contribué à la victoire par soldats interposés, et sa valeur rend légitime la gloire qu'il en retire.

L'épopée traditionnelle, qu'il s'agisse des poèmes homériques ou de la chanson de geste, renvoie à une époque éloignée et souvent mythique, en tout cas à une époque qui se situe au-delà de l'expérience du poète; le poème de combat qui raconte un événement récent inscrit l'action dans un temps vécu. Alexandre de Pontaymeri, un protestant modéré favorable à Henri IV, indique qu'il a composé La Cité de Montelimar rapidement, dans l'émotion que l'événement a provoquée:

Amy lecteur, si je te communique ce mien ouvrage, esbauché parmy les feus des guerres civiles, le brazier des assauts, et la sanglante poussiere des combats, je te prie de croire que ce n'a point esté pour me faire voir au theatre de ce monde, où ceux qui jouënt des calomnies populaires: mais que seulement la verité princesse unique de mes affections, m'a dicté ceste histoire, marque éternelle de la valeur de ceux, au service desquels j'honore l'estat de ma vie: sans rien adjouster aux divers evenements de la guerre, qui est comprise en ce cayer, où je suis totalement historien contre la nature de tous les poëtes: je dy en ce qui est des principales matieres. Car pour quelque legere invention que je mesle parmy, il n'est pas raison que tu conclues de l'universel par le particulier. Aussi je m'en remets à ta modestie, te protestant que tout tel qu'est ce mien œuvre, je l'ay conceu et enfanté en un seul mois. Bref, c'est le crayon de mon ame, qui ne souspire que la mesme impatience: car je te jure que l'original qu'en a eu l'Imprimeur, n'est que le simple brouillard de mes premieres conceptions touchant ce livre $[\ldots]^{7}$.

Ce texte éminemment révélateur présente le récit de bataille comme un témoignage et exprime la continuité entre la composition poétique et l'action guerrière. L'absence de rupture garantit la vérité du discours, car elle implique respect des héros du jour et fidélité aux événements. Pontaymeri rappelle l'opposition aristotélicienne entre le poète et l'historien et revendique résolument le second statut. Le poème ne naît pas de la fureur poétique, mais de l'impatience. On pourrait rapprocher cette impatience du calor subitus, cette chaleur spontanée, incontrôlée, qui selon la préface du premier livre des Silves de Stace, s'empare des poètes. Le calor renvoie à une conception de l'inspiration moins élevée, plus humaine, que le furor. Il est l'effet d'une activité intense de l'esprit, qui naît d'une émotion sincère (sympathie, admiration, gratitude), de l'improvisation et d'une durée de composition resserrée. Il préside notamment à l'éclosion de pièces de circonstance que, dans un second temps, la conscience critique organise et amende ${ }^{8}$.

(6) P. DE Deimier, 1601, f. a2 vº.

(7) A. DE Pontaymeri, 1591, «Au lecteur», p. 5. Sur A. DE PONTAYMeri, voir B. MÉnIEL, «Écrire noblement au XVI siècle: les choix formels d'Alexandre de Pontaymeri» in Le Statut littéraire de l'écrivain, Travaux de Littérature, XX (2007), pp. 247-268.
(8) Voir P. Galland-Hallyn et F. Hallyn, dir., Poétique de la Renaissance. Le modèle italien, le monde franco-bourguignon et leur béritage en France au Xvie siècle, Genève, Droz («Travaux d'Humanisme et Renaissance»), 2001, pp. 132-134. 
Lorsqu'il adresse au duc de Mercœur un poème sur la bataille de Craon, Nicolas de Montreux déclare dans sa préface:

Or Monseigneur, m'estant veu de tout inutile à vous servir, en ceste vostre glorieuse entreprinse. J'ay pensé, que je devais pour le moins l'escrire. Je l'ay faict, non en esperance de dignement m'en acquitter. Car je croy que le grand Homere y feroit paine. Mais pour me jecter au rang de tant de doctes ames, qui vous esliroit pour digne subject de leurs escrits. Aussi que je vous suis lié de pareille servitude, et vive obligation que Virgile à Cesar. J'ay donc escrit cest heureux discours en vers, que sçay vous estre aggreables, pour estre autant excellent ouvrier d'iceux, que Poëte que nous ayons, et plustost qu'en prose. Parce que j'ay pensé, qu'un chant de victoire, se devait chanter en vers mesurez, et non lire en prose. Il s'y trouvera fort amplement descrit, en l'histoire des actes memorables de Bretagne, dont vostre grandeur m'a tant honoré que me charger. Pendant voyez cest avancoureur, qui m'est plus doux qu'à vous-mesmes, bien qu'il ne souspire que votre los, pour me veoir si fortuné, que de chanter la victoire du plus grand et victorieux Prince qui vive?

N'ayant pu servir son maître par les armes, Montreux le sert par la plume. Encore dans l'émoi du succès de son camp, il compose un «discours» écrit «en vers», un «chant de victoire» «avantcoureur» de «l'histoire des actes de Bretagne»: une complémentarité se dessine entre un poème relativement bref, né de la réaction affective à l'événement, et un récit historique en prose plus développé et rédigé avec recul et pondération. Montreux s'inscrit dans une double tradition: celle du poète de cour, dont Virgile constitue le modèle; celle du poète-orateur, dont l'éloquence épidictique trouve à s'investir aussi bien dans des pièces de circonstance que dans l'historiographie.

Contrairement à l'usage dans la poésie épique, l'auteur de récit de bataille recourt volontiers à la première personne; il exprime parfois même les sentiments que l'événement a provoqués en lui. Par exemple, Alexandre de Pontaymeri ne cache pas son émotion lorsqu'il évoque la honte que suscite le massacre qui aboutit à la reprise de Montélimar par les troupes royales:

Les vivants et les morts sont en nombre pareil,

Le Ciel en est honteux, honte en a le Soleil,

$[\ldots]$

Moy-mesme en suis honteux, et mon riche cayer

Se vermeiIle en leur honte, à noircir coustumier ${ }^{10}$.

L'éthos du poète de circonstance se construit dans un refus à la fois de l'équanimité de l'historien et de l'effacement du poète épique.

La bataille est donc considérée à hauteur d'homme. Le poème est dédié à un Prince ou à un grand capitaine qui a participé à la bataille: le premier destinataire est le héros du jour. Chez Pontaymeri, le poème dans son ensemble est dédié à Lesdiguières, et chaque livre à un de ses lieutenants. Si l'on prend au pied de la lettre cette situation d'énonciation, la fonction du récit de bataille en vers ne peut être d'informer, car il serait absurde de vouloir enseigner les détails de l'événement à celui qui en a été le premier acteur.

La présence d'embrayeurs temporels distingue radicalement l'epyllion de victoire du poème héroïque évoquant un passé lointain et mythique: il n'y a pas de

(9) N. DE Montreux, 1592, épître dédicatoire, f. A ij $v^{\circ}-A$ iij $r^{\circ}$.
(10) A. De Pontaymeri, 1591, VI, p. 208, vv. 2021; p. 209 , vv. $4-5$. 
césure entre le temps de l'énonciation et celui de l'histoire; la narration s'inscrit dans la même temporalité que l'action et la prolonge.

Cet écart par rapport à la tradition épique n'a guère d'incidence sur la façon dont l'événement est reconstruit. Fondamentalement, le récit de bataille emprunte à l'epos sa structure moléculaire: se succèdent, sur le mode de la parataxe, un certain nombre de séquences narratives, dont certaines, déjà identifiables dans l'Iliade ou dans la chanson de geste, ont acquis le statut de motifs. Prenons par exemple La Cité de Montelimar d'Alexandre de Pontaymeri. Les livres V et VI racontent l'attaque victorieuse lancée par les troupes royales contre les ligueurs qui tiennent la ville. Le livre V présente une revue des troupes (ou epipôlèsis), une vision du théâtre des opérations du haut d'une tour (ou teichoskopia), une harangue, un serment (le capitaine des troupes royales se jure de ne prendre aucun repas avant d'avoir regagné sa demeure), un conseil, un signe céleste et son interprétation par un vieillard, un duel. Le livre VI propose les encouragements que le chef prodigue à ses troupes, l'armement, la prière, l'attaque des enfants perdus, puis celle du gros des troupes, les exploits individuels (ou aristeiai), les morts et les lamentations, une descente aux enfers, les monceaux de morts et les ruisseaux de sang, la déroute. Ainsi le poète ne connaît pas d'autres moyens de rendre compte d'un événement réel que le recours à des motifs millénaires. La contradiction est évidente: d'une part il s'adresse au capitaine vainqueur et il témoigne sur une bataille singulière, dont il s'efforce de dégager les particularités; d'autre part, il recourt à des stéréotypes, qui informent et déforment l'action et substituent à la succession des faits l'agencement de séquences narratives traditionnelles.

Nous ne pouvons lever cette contradiction qu'en nous interrogeant, à partir d'un exemple précis, sur la fonction du motif. Lorsque Sébastien Garnier, au livre XVI de la Henriade raconte comment à son retour de la bataille d'Ivry, Henri IV est accueilli par la liesse de ses troupes, il emploie un motif traditionnel, le retour du combat, et il fait explicitement référence à un passage homérique:

La joye que jadis receurent les soldars,

Arrangez sur les murs et furieux rampars

Du superbe Illion, qui regardoient combattre

Leur Hector contre Ayax, tachans à s'entr'abattre,

Le voyans sain et sauf du combat de retour,

Me fut acomparer à l'aise que ce jour

Receurent noz soldars, de revoir retournee,

La personne du Roy ceste belle joumee,

Sans avoir aucun mal, et le voyant venir,

Plusieurs ne se pouvoient de joye contenir

De plorer tendrement, espouvantez de crainte

Qu'il n'eust en combattant quelque facheuse attainte $[\ldots]^{11}$.

Le poète indique en manchette sa source: le combat d'Hector et d'Ajax au septième livre de l'Iliade [vv. 307-310]. Il assume donc, et même revendique, la reprise de motifs antiques. Il instaure ainsi une relation intertextuelle, qui n'ajoute rien à la véracité et à la précision du récit et qui atteste au contraire l'intervention de médiations littéraires. Une telle démarche indique clairement que le poète n'est pas fidèle à l'événement de la même façon que l'historien: il ne s'efforce pas de raconter dans le détail les phases de la bataille ou de reconstituer la chaîne de causes et d'effets qui expliquerait son issue, mais plutôt de donner sens à l'événement au moyen d'une élaboration symboli- 
que. L'emploi de motifs archétypaux signale donc l'accès à l'héroïsme, à la grandeur, à la gloire. Les guerriers d'Ivry ne sont pas indignes de ceux de Troie. Ce que le récit de bataille perd en exactitude référentielle, il le gagne en sacralité. La stylisation est un effort pour s'arracher à l'emprise du réel et s'élever au plan des significations.

La forme épique n'est donc pas choisie pour sa souplesse, pour la capacité qu'elle aurait de rendre compte avec fidélité du déroulement des faits. Elle est au contraire adoptée parce qu'elle met l'événement à distance et qu'elle en donne une représentation stylisée et signifiante.

Le récit de bataille est le moyen d'appréhender un événement. Or qu'est-ce qu'un événement? C'est ce qui introduit une rupture dans la continuité historique, c'est l'inattendu ou le trop longtemps attendu, l'imprévisible, l'impensable. Le seul moyen de penser l'impensable est d'en rendre compte au moyen de formes connues. A cette fin, l'épopée peut prêter sa forme. En particulier, elle implique un rapport à l'espace et au temps, un chronotope, comme dirait Bakhtine. Peut-être l'étude de ce chronotope nous permettra-t-elle de percer les intentions des poètes qui usent de la forme épique pour célébrer des exploits militaires récents.

\section{Un rapport à l'espace et au temps}

L'epos chante les phénomènes. Il a l'ambition de saisir l'histoire dans son avènement. D'où son sens du concret, son attention au monde des choses. Il se réalise pleinement lorsqu'il donne au lecteur l'illusion d'être en présence de ce qu'il évoque. C'est la figure de l'hypotypose. Dans l'Austriade, Quirin et Canal ont déclenché un incendie dans les galères turques grâce à des flèches enflammées:

Ces chevrons enflamez, lancez d'art et de force,

Et portez plus legers par la flambante amorce,

Dans les barques des Turcs allumaient des brasiers

Non moins pernicieux qu'audacieux et fiers:

Ils embrasaient la caisse, et les rangs et la poupe,

Cordes, arbre et antenne, et redoublant leur troupe

Ils se lançoient aux flancs, où tendus à la poix,

Ils s'acharnoient apres à l'espaisseur du bois:

On voyait peu à peu leur puissance plus grande,

Et luire plus cruelle aux flancs et à la bande,

Et consommer le bois avec un craquement,

Qui bruyant dans le feu couroit obliquement.

On voyoit blueter sous la noire fumiere

Les flambeaux devorans d'une horrible lumiere.

Ces lieux par ces nuaux espaissement noircis

Estoient du feu d'en bas ardans et esclaircis,

Le vent qui tourmentoit ceste flame indomtée

La rendait çà et là plus fiere et agitée ${ }^{12}$.

Le poète fait pour ainsi dire pénétrer le lecteur dans l'espace de l'action, au moyen de présentatifs, des déictiques, d'adverbes de lieu («çà et là»), du présent de l'indicatif, de verbes de sensation («on voyoit»), de fragments de description limités à des indica- 
tions suggestives des matières, des formes et des couleurs. A un tableau panoramique, il préfère une description particularisante, qui procède par petites touches et pratique l'énumération.

L'epos ne se contente pas de mettre sous les yeux l'espace du combat, il en propose aussi, souvent, une vision surplombante. Un de ses procédés emblématiques est la teichoskopia, ou vision depuis les remparts. Dans La Cité de Montelimar, la teichoskopia est le fait d'une allégorie: l'Effray «cyclope carnacier vivant d'occision» (p. 132, v. 6) se perche sur la plus haute tour de la ville, observe les soldats ligueurs qui murent le château, et répand parmi eux le mépris du devoir, le dédain de la vie, la faiblesse, l'imprudence... De même, pour offrir une vue d'ensemble du combat naval de Lépanthe, Deimier imagine un berger observant la scène de loin:

Lors un Pasteur planté sur la cime d'un Mont,

La fumée à longs plis voit s'envoler à mont,

Et puis il voit en bas ces escadres diverses,

Presser si grand païs sur les campaignes perses,

Et tant de vistes feux sur les bronses fumans,

Il oit les ton-tonnans des boulets escumans,

Et voit les flots salez blanchir en estincelles,

Par la prompte carene et les rames isnelles:

Puis il voit ja desja quelque gaillard vaisseau.

S'acrocher et se batre en un combat nouveau,

Tandis qu'en autre part le canon qui poudroye

Encore d'un peu loing ses tempestes foudroye.

Lors d'un tel spectacle esmeu dedans son cœur,

L'effray luy peinct le front d'une blesme langueur,

Le poil luy dresse au chef, il demeure immobile,

Comme les membres froids du rocher de Sipyle,

De mesme peur ateinct ses chiens et ses toreaux,

Sans courir, sans manger, tiennent bas les naseaux,

Et cent fois ce Pasteur louë son sort rustique,

Sus la guerre qu'il voit en si rude pratique ${ }^{13}$.

Au motif du spectateur surplombant se superpose le topos du suave mari magno. Comme l'hypotypose, la teichoskopia permet d'apprivoiser l'espace.

Sur la succession des moments s'exerce une vigilance plus grande encore. La temporalité dans laquelle Pontaymeri inscrit son poème est celle de l'Histoire, à la chronologie précise. La bataille se déroule dans une durée close où se jouent des destins et où s'achète l'honneur. Le rapport au temps est donc fortement dramatisé. En particulier la nécessité d'entreprendre l'action opportune est mise en avant. Cette poésie d'occasion qu'est le récit de bataille en vers célèbre l'aptitude du chef de guerre à saisir le moment opportun. Le kairos poétique rejoint le kairos tactique. Dans la Reduction de la ville de Bone, un élément infime décide de la victoire: certains défenseurs de la ville, qui occupent un fort d'importance stratégique, se révèlent disposés à traiter; c'est l'occasion que le duc de Croy, lieutenant d'Alexandre Farnèse, ne laisse pas passer:

Car advint tot apres par une chance adverse

De l'estrange Bellonne, en sa course diverse,

Qu'un nombre d'Allemans, dedens l'aile posez, 
Se monstrent de traiter humblement disposez,

De ceste aisle apointant: Aisle tant importante,

Que la force du cœur, sans elle, n'est bastante

D'emprendre un lointain vol: heureuse occasion,

Qui submit tout le fort à la devotion

Du Prince gracieux, à fin qu'en fait semblable

Bone, bonne se feit, et non pas miserable,

Prudemment eslisant un honneste party $[\ldots]^{14}$.

Dans La Cité de Montélimar, alors que les troupes de Lesdiguières frémissent du désir de libérer la ville, le sieur de Blacons s'adresse à Le Poët, pour l'inviter à saisir l'occasion:

«L'occasion est chauve, et qui ne la retient

«Tout soudain elle eschappe et jamais ne revient $[\ldots]^{15}$.

Les poètes recourent aux formules que leur lègue une sagesse ancestrale: Érasme cite à propos de la déesse Occasio le beau vers: Fronte capillata est, post haec Occasio calua $^{16}$, et repère l'expression capere crines dans Mostellaria de Plaute ${ }^{17}$. Le présent gnomique, les verbes d'obligation signalent le discours parénétique. Le récit de bataille ne s'adresse donc pas à des citoyens passifs dont il ne requerrait que de l'admiration. C'est un genre militant qui change son lecteur en guerrier. Il entend influer sur son comportement en proposant des principes d'action, des modèles à imiter, un concentré d'expérience, comme l'atteste la place qu'il accorde au discours éthique sur l'occasion.

Certes, l'événement s'insère dans une chronologie précise et particulière qui le sépare du mythe; le poète le rattache néanmoins à celui-ci par un travail de sacralisation qui l'inscrit dans un autre rapport au temps. En multipliant les signes du soutien céleste, tels que ces coups de tonnerre qui manifestent l'appui apporté au grand capitaine, il fait de la bataille le point où se concentre l'action providentielle de Dieu. Le récit de l'événement apparaît comme le moyen de saisir l'Histoire en train d'advenir.

Toujours la victoire apparaît comme un signe de la coïncidence de l'action humaine avec le dessein de Dieu; le Prince qui triomphe est un élu du Ciel:

C'est un des plus grands biens, et digne recompence

Que l'on pourrait avoir: à celui seul est deu

Que le grand Dieu d'enhaut a choisi et esleu,

Qui parfaict et entier, et net de conscience,

Remet du tout en luy, sa devote esperance.

$\mathrm{O}$ trois et quatre fois, ô Prince valleureux

Par sus tous Roys vivans, et Monarques heureux,

Roy bien aymé de Dieu, le Ciel, la Terre et l'onde,

Combattent pour ton nom, brief tout l'univers monde $[\ldots]^{18}$.

La divinité tutélaire qui préside au succès militaire est le «Dieu des armées», le «Dieu des victoires»:

(14) J. Bosquet, f. A3 vº, v. 17; A4 r ${ }^{\circ}$, v. 3.

(15) Ibidem, IV, p. 115, vv. 13-14.

(16) ÉRASME, Adagiorum chiliades quatuor cum sesquicenturia [...], Paris, Nicolas Chesneau, 1579, adage Nosce tempus (1.7.70; col. 232): «Elle a des cheveux sur le front; derrière, l'Occasion est chauve».

(17) Ibidem, adage Capere crines (4.10.39; col. 949). Cfr. Plaute, Mostellaria, v. 226.

(18) S. GARnIER, 1593, XIV, p. 105, vv. 6-14. 
celuy vainc tousjours

Qui n'attent d'autre part que de Dieu son secours,

C'est le Dieu des combats, sa main et non les armes

Rend deffaicts ou vaincueurs les orguilleux gendarmes ${ }^{19}$.

La poétique implicite du récit de bataille est donc remarquablement cohérente. Entre la situation d'énonciation, la structure, le chronotope, existe un accord profond, qui n'est cependant pas celui qui fonde le poème épique traditionnel: ni l'espace, ni le temps de l'action ne sont coupés de ceux dans lesquels évoluent le poète et le lecteur. Tout est fait au contraire pour que le lecteur soit ému, et qu'il ait jusqu'à un certain point l'illusion de prendre part à l'événement raconté. Ce pathos devrait permettre de déterminer les fonctions du poème.

\section{Les fonctions du poème}

Dans l'epyllion de victoire, le poète adresse des remerciements à Dieu, notamment dans l'invocation, Ainsi, Nicolas de Montreux présente explicitement son poème sur la victoire de Craon comme une action de grâce:

Reçoys donc ô bon Dieu ce pieux sacrifice,

Cest action de grace à ta bonté propice,

Que l'odeur en soit douce, et ne permets pieux

Que nous soyons chantez ingrats par noz nepveux ${ }^{20}$.

Comme l'hymne ou le cantique, l'epyllion de victoire s'adresse à Dieu pour exprimer la reconnaissance de la communauté. Cependant, l'hymne et le cantique maintiennent de bout en bout la même posture, alors que l'epyllion l'abandonne après les premiers vers: dans la narration, le poète tend à s'effacer, même s'il reste davantage présent que dans le poème héroïque, et il n'apostrophe Dieu que rarement. La narration dans sa totalité célèbre l'action divine et il n'est pas nécessaire de multiplier les marques de gratitude. En général, Dieu n'est l'allocutaire que dans l'invocation et il devient dans la narration un personnage à part entière. Ainsi, au dixième livre de la Henriade, Sébastien Garnier fait intervenir Dieu: un cordelier envoyé par le légat pontifical, le Cardinal Cayetan (Cajetan), encourage la troupe des Ligueurs, dont il prend tous les péchés sur la conscience; ne pouvant supporter cette injure, «le grand

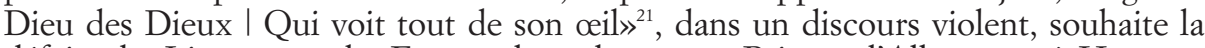
défaite des Ligueurs et des Espagnols, ordonne aux Princes d'Allemagne, à Henry et à Elizabeth de faire le nécessaire et envoie l'Archange Michel pour qu'il expédie les Ligueurs aux Enfers. Ainsi, l'epos témoigne de la toute-puissance céleste, mais il rend grâce, tout autant qu'à Dieu, aux hommes qu'Il soutient.

A la fonction de remerciement s'ajoute celle de commémoration. Selon un topos que développe souvent l'epyllion de victoire, il revient aux poètes de dispenser la gloire: sans eux, les exploits passés seraient oubliés ${ }^{22}$. Le récit de bataille en vers indique aussi que le succès dépend pour l'essentiel de la vaillance des chefs:

(19) N. De Montreux, 1592, f. D r ${ }^{\circ}$, v. 28 ; $\mathrm{v}^{\circ}$, v. 1.

(20) Ibidem, f. Bij r ${ }^{\circ}$, vv. 21-24.
(21) S. GARNIER, 1593, X, p. 25, vv. 29-30.

(22) N. De Montreux, 1592, f. Biij vo. 
De la valleur du chef provient toute la gloire,

Seul du bras ennemy il ravist la victoire,

Car vaillant comme luy il rend des siens le cœur,

Et l'ennemy craintif au feu de sa valleur,

Le soldat ne combat, brave, que par exemple,

Si son chef est vaillant, vaillant on le contemple.

Mais si son chef s'enfuit, il fuit encore mieux,

Les lions sont des serfs, conduicts d'un serf poureux ${ }^{23}$.

L'esthétique de l'épopée, qui concentre l'attention sur les chefs, s'accorde donc avec la conception aristocratique de la guerre au $\mathrm{XVI}^{\mathrm{E}}$ siècle, qui, dans le succès militaire, attribue aux capitaines un rôle incommensurablement plus important qu'à la troupe. Le poème doit perpétuer le nom et le renom des guerriers valeureux. Montreux le dit clairement:

Et que là peinct on voye en un tableau de cuivre,

Et le nom de mon Prince, et ceux qui pour le suivre

Ont cousu le harnois aguerry sur leur dos,

Et ceux qui ont chanté fidellement son los $[\ldots]^{24}$.

Face à la postérité, les hommes de guerre et les hommes de lettres sont unis par un même destin. Comme une des missions essentielles que se donne l'epyllion de victoire est d'immortaliser les héros du jour, son discours épidictique assigne au nom propre un rôle-clef. A la fin de son poème, Pontaymeri dresse une liste des seigneurs prisonniers; mais il l'écourte bientôt, car il ne souhaite pas assurer à tous l'immortalité:

[...] Mille et mille autre encor, que mon plaintif cayer

Ou ne doit ou ne veut chez le temps envoyer.

Le temps qui fauche tout: Car tous ceux que je nomme

Triompheront du temps et de la mort, en somme.

Les vainqueurs de ceux-cy mesme seroient vaincus

Par la mort et le temps, et seroient incognus

Sans moy qui fay broncher sous le pois de leur gloire

Le present, le futur au laps de ma memoire

Qui triomphe du temps: Car son antiquité

Appelle mes escris enfans d'eternitée 25 .

La fréquence des noms propres est parfois considérée par les poétiques de la Renaissance comme un trait caractéristique de l'historiographie, qui introduirait de la rudesse dans la poésie: «[...] la rudesse des noms propres qui affièrent à l'histoire, ne trouve pas facilement place dedans la composition sujette à mesure, comme sont les vers $\gg^{26}$. Au contraire le poète qui compose un récit de bataille s'appuie sur les patronymes et les titres des combattants. Ceux-ci sont souvent placés en fin de vers, de sorte que l'exercice s'apparente à un bout-rimé. Nicolas de Montreux s'adresse au Duc de Mercœur en ces termes:

(23) Ibidem, f. I v ${ }^{\circ}$, v. 28, Iij $\mathrm{r}^{\circ}$, v. 3.

(24) Ibidem, f. Bij vo, v. 32, Biij ro, v. 2.

(25) A. DE Pontaymeri, 1591, VII, p. 229, vv. $15-24$.

(26) J. Peletier, Art poétique (1555), I, 3, «Du
Sujet de Poésie: et de la différence du Poète et de l'Orateur», in Traités de poétique et de rbétorique de la Renaissance, éd. Francis Goyet, Paris, Librairie générale française («Le Livre de poche classique»), 1990, p. 232. 
Alors tu faits marcher le Marquis de Bel-isle,

Brave comme un Cesar, vaillant comme un Achille,

Talhoüet quant et luy, brave experimenté,

Tous les chevaux legers à leur masle costé,

Ils chargent vivement, avec eux Chevriere,

Les Loges, qui menoit ceste bande guerriere

De soldats à cheval du vaillant Bois-dauphin,

Qui demeure immortel en despit du destin $[\ldots]^{27}$.

Deimier tire parti des sonorités des noms exotiques. Certains vers de l'Austriade ne sont plus qu'une accumulation de noms de guerriers:

Mais Don Jean, le Cesar dont l'Espaigne se dore,

Suivit de Benevide, et de Pierre Sapore,

De Louis de Cardonne ame de mesme ardeur,

Et du vaillant guerrier l'Espaignol commandeur,

De Ferrier et Sotto, Rhedie et Figuarée,

De Gusmane et la Plegue à la force hectorée,

De Bernard de Cordouë, et de Moncade encor',

Et du preux Salasar, d'un cœur esgal à l'or

Espurgé par sept fois dans la purgeante flame,

Vint donner courageux, de front, de main et d'ame,

Sur les Turcs $[\ldots]^{28}$.

Dans les énumérations, aux noms espagnols s'ajoutent les noms turcs:

Bayram, et Orman et Altonte les forts,

Sous le fer de Moncade avaient fini leurs sorts.

Le fameux Commandeur de la noble Castille

Avoit tué Vely, Deniram et Hortille,

Et le hautain Sigsiam. Le vaillant Salasar

Avait mis sur le champ Gusel, Humer, Falsar,

Et le fier Baialbam. Le genereux Sapore,

Corcut, Arsam, Zathnir, Stambolquir et Pandore ${ }^{29}$.

Lorsqu'elle prend la forme épique, la poésie de circonstance se constitue en monument.

Le souci des noms propres procède de l'attention portée à l'individuel et au particulier. Chez les poètes ayant assisté aux événements racontés, des détails concrets, parfois incongrus, en tout cas inutiles au propos épidictique, créent un effet de chose vue. Dans La Cité de Montelimar, l'invincible Beaulaigne tue un capitaine, puis Adail, Teronnet et Pisin. D'un coup de hache, il évide les orbites d'un ennemi:

Le coup fut nompareil: car sans meurtrir la teste,

Et sans froisser le casque, a la glissante creste

La hache artistement luy cerna les deux yeux,

Les prunelles en haut jaillirent vers les Cieux,

Ses genoux tremblotans, et ses mains remuantes

Demonstroyent la rigueur de ses peines cuisantes,

Du crespelé menton il feit signe troi-fois

(27) N. De Montreux, 1591, f. Iij v

(28) P. De Deimier, 1601, I, p. 53, vv. 1-11.

(29) Ibidem, I, p. 57, vv. 1-11. 
Comme s'il eut voulu r'articuler sa voix,

Redifier son coeur, et reunir sa force

Lorsqu'il tomba surpris d'une mortelle entorse ${ }^{30}$.

Le recours à des termes laudatifs («nompareil», «artistement») surprend dans l'évocation d'un meurtre cruel. Pourtant, la gratuité du geste et la précision clinique de la description signifient l'authenticité du témoignage: de tels passages rapprochent l'esthétique du poème de combat de celle des mémoires. Ils sont rares, cependant, et la plupart du temps, le poète gomme les détails trop particuliers et use de motifs traditionnels, afin de conférer à l'événement une grandeur mythique: sous sa plume, l'affrontement décrit devient la bataille par excellence. Comme dans un palimpseste, à travers le récit de tel affrontement réel transparaissent des schèmes très anciens.

La commémoration n'est pas seulement enregistrement de ce qui a été accompli; elle est incitation à imiter les exploits racontés et à poursuivre le combat: le récit de bataille mobilise. Peletier du Mans écrit par exemple:

La Poésie a été cause que les faits mémorables des hommes illustres, sont passés à la postérité. Et rendant témoignage de la grandeur et excellence des vertus et vertueux: a fait que les suivants se sont efforcés à semblables factions et entreprises, pour le désir de l'immortalité: et que pour le salut et conservation de la patrie, les hommes vaillants ont exposé leur propre vie: premièrement pour être honorés de leurs hommes et pour avoir la faveur de leurs Princes: puis après, pour être perpétués par la guide faveur de leurs Princes: puis après, pour être perpétués par la guide des Écrits ${ }^{31}$.

La visée à long terme - la gloire - est indissociable d'une visée à court terme - l'action politique.

Deux motifs plus que tout autre indiquent les enjeux de la bataille: la prière que profèrent les femmes, la troupe ou le chef militaire, et la harangue que prononce le général avant l'affrontement. Ces deux types de discours rapportés permettent de définir les valeurs au nom desquelles la lutte est menée. Dans la Henriade de Garnier, le personnage d'Henri IV fait, avant que la bataille d'Ivry ne commence, une émouvante oraison où il se présente comme un roi stoïcien, entièrement soumis à la Providence ${ }^{32}$. Dans La Miraculeuse Délivrance de Monseigneur le Duc de Guise de Montreux, le Duc de Mercœur galvanise ses hommes en leur rappelant qu'ils combattent pour 1'honneur de Dieu et pour la liberté:

Nous ne combattons pas seulement pour la vie,

Pour nos biens, nos maisons, et pour nostre patrie,

Mais pour l'honneur de Dieu, pour nos ames aussi,

Qu'un tiran orgueilleux veut forcer sans mercy $y^{33}$.

Il ajoute même le salut de la patrie. Il place ces valeurs plus haut que le simple sens de l'honneur et que l'appétit de conquête:

Si l'honneur seulement eut le pouvoir jadis

D'armer nos peres vieux, et les rendre hardis

Contre leur ennemy, si jadis pour la terre

On a veu tant de Rois s'entre-faire la guerre,

(30) A. De Pontaymeri, 1591, VI, p. 203, v. 23; p. 204, v. 7.

(31) J. Peletier, op. cit., I, 1, «De l'antiquité et de l'excellence de la Poésie», pp. 225-226.
(32) S. Garnier, 1593, IX, p. 16, v. 19; p. 17, v. 14, texte cité et commenté dans notre Renaissance de l'épopée, éd. cit., pp. 364-365.

(33) N. De Montreux, 1591, f. Hiiij rº, vv. 8-11. 
Combattre sans repos, et rendre mille fois

Les champs semez de sang, de corps, et de harnois.

Combien heureusement, et d'une masle audace

Devons nous aujourd'huy combattre en ceste place?

Où l'honneur du grand Dieu, la foy, la liberté,

Et le salut du pais, se voirra disputé $e^{34}$ ?

Parfois, le poète apostrophe le lecteur pour l'exhorter à tirer les leçons de la victoire et à défendre le parti qu'elle conforte. La Vallée du Maine invite le peuple à embrasser la cause ligueuse:

[...] Dieu le tout-puissant, monstre par son effect,

Qu'il est le Roy des Roys, seul entier et parfaict.

Donc peuple renge toy, à sa seulle puissance,

Cest luy seul qui pourra, te delivrer d'outrance,

Invocque son Sainct nom, pour les Princes Lorrains

Qui te vont delivrant, des bourreaux inhumains,

Prie pour les Seigneurs, qui de vaillant courage

Te veullent delivrer, de fureur et de rage $[\ldots]^{35}$.

L'indicatif cède le pas à l'impératif. Les exploits passés ne sont relatés que pour encourager les actions à venir.

Les récits de bataille que nous avons étudiés sont donc nés à la jonction d'une occasion - une bataille, qui est le plus souvent une victoire -, d'une intention - l'action politique -, d'un genre rhétorique - l'épidictique - et d'un genre poétique - l'épique. Leur dynamisme résulte de tensions immédiatement sensibles: entre la poésie et l'histoire, entre le motif stéréotypé et le témoignage, entre la louange hyperbolique et la vérité impartiale.

Notre analyse confirme qu'un genre littéraire n'est pas seulement une forme, ni même un ensemble de traits stylistiques, c'est un rapport au monde, et dans le cas présent, une relation à l'Histoire. Un même rapport à l'Histoire unit le grand capitaine et l'écrivain. La poésie de circonstance n'exprime pas une fascination devant la poussière de l'événement, devant les miettes de l'actualité, mais l'insertion d'un fait dans l'éternité de l'Histoire. Cette poésie est attentive au moment présent, à sa charge émotive, à la capacité d'action qu'il offre, mais elle est aussi consciente de sa responsabilité face à la postérité. Le poète, lui aussi, fait l'Histoire.

BRUNO MÉNIEL 